\section{Estresse e função reprodutiva feminina}

\section{Stress and female reproductive function}

Simone da Nóbrega Tomaz Moreira 1 Josivan Gomes de Lima 2

Maria Bernardete Cordeiro de Sousa 3 George Dantas de Azevedo 4
Abstract This article discusses the relationship between stress and reproduction considering that infertility could bear psychological causes (psychogenesis hypothesis) or could be caused by psychological stress. Stress has the potential of activating the hypothalamus-hypophisis-adrenal axis inhibiting the hypothalamus-hypophisis-ovarian axis leading to temporary menstruation paralysis. This process could result in a transitory infertility of women. The authors emphasize the need of a psychological approach in reproduction services with the objective of treating tensions and frustrations derived from infertility and its treatment.

Key words Reproduction, Stress, Infertility, female, Psychology
Resumo Este artigo discute a relação do estresse sobre a função reprodutiva, considerando que a infertilidade pode ter causas psicológicas (hipótese da psicogênese) ou pode ser a origem do estresse psicológico. A presença do estresse tem o potencial de ativar o eixo hipotálamo-hipófise-adrenal, o qual, inibe o eixo hipotálamo-hipófise-ovariano, levando à paralisação temporária das menstruações. Esse processo pode resultar em infertilidade transitória para as mulheres. Os autores enfatizam a necessidade de uma abordagem psicológica nos serviços de reprodução, objetivando trabalhar as tensões e frustrações advindas da infertilidade e do seu tratamento.

Palavras-chave Reprodução, Estresse, Infertilidade, feminina, Psicologia 


\section{Introdução}

Há décadas diversos autores têm destacado o estresse como fator desencadeante de alterações na função reprodutiva. Nesses trabalhos iniciais já se considerava a possibilidade de um evento estressor, conhecido ou latente, inibir o eixo hipotálamo-hipófise-gonadal e acarretar irregularidade menstrual, amenorréia e infertilidade. ${ }^{1}$ Entretanto, a influência dos estados psicológicos sobre a função reprodutiva apresenta um perfil multifatorial, sendo muito difícil, senão impossível, determinar relações lineares de causa e efeito.

O impacto que a condição psicológica tem sobre o organismo é determinado por uma combinação de fatores, tais como: traços de personalidade, presença ou não de transtornos mentais, disponibilidade de suporte social e estratégias de adaptação diante de uma situação estressante. Dessa forma, a resposta endócrina aos estímulos ambientais depende de variáveis biopsicossociais. 2 Além disso, a reação endócrina ao estresse é dependente do traço de ansiedade, sendo observadas respostas hormonais mais intensas em indivíduos portadores de maior nível de ansiedade.3,4

Vários pesquisadores utilizaram o "Inventário de Ansiedade Traço-Estado (IDATE)" para avaliar em que medida essa variável psicológica pode influenciar o eixo hipotálamo-hipófise-ovariano. Observações apontam que quanto maior o nível de ansiedade, menor a chance de gravidez em mulheres submetidas à inseminação artificial.5-7 Por outro lado, diferentes transtornos mentais têm sido associados a alterações do ciclo menstrual e reprodutivo, tais como transtorno de estado de ânimo, transtornos adaptativos e transtornos de conduta alimentar. 8 Percebe-se que a base funcional da resposta ao estresse participa de todos esses transtornos e que as reações neuroendócrinas decorrentes podem comprometer a função reprodutiva, sendo esses efeitos mediados por interferências dos hormônios relacionados ao estresse sobre o eixo hipotálamo-hipófise-ovariano.

O estresse é capaz de interferir na função reprodutiva em diversos aspectos. Na ausência de uma causa orgânica detectável, a supressão dos eventos normais do ciclo reprodutivo é chamada de amenorréia hipotalâmica funcional ou de anovulação crônica hipotalâmica funcional. Essa anormalidade está relacionada com estresse físico e emocional, como ocorre nos casos de perda excessiva de peso, anorexia e exercícios físicos extenuantes (amenor- réia da atleta); nesses casos, alterações no balanço metabólico parecem ser a causa principal. Esse artigo tem como objetivo destacar, especificamente, as influências do estresse sobre os diversos mecanismos de regulação da função reprodutiva em mulheres.

\section{Eixo hipotálamo-hipófise-adrenal (HHA)}

A resposta endócrina ao estresse psicológico tem sido extensivamente estudada, tanto em animais quanto em humanos. Analisando marcadores do estresse em humanos, ${ }^{4}$ pesquisadores afirmam que a relação entre intensidade do estresse e a resposta endócrina é pouco conhecida. Todavia, a partir de resultados obtidos com roedores, observou-se que estressores físicos ou emocionais atuam sobre o hipotálamo alterando a secreção de fatores liberadores ou inibidores de hormônios hipofisários. Nessas situações, a liberação do $\mathrm{CRH}$ promove aumento da produção do hormônio adrenocorticotrófico (ACTH), o qual, por sua vez, estimula a ativação do sistema simpático, com liberação de cortisol e catecolaminas (Figura 1), responsáveis pela resposta fisiológica ao estresse. ${ }^{9}$

Pesquisas experimentais demonstraram que estressores físicos e psicológicos produzem um imediato e constante aumento nas concentrações de CRH.9,10 Além dos aumentos do ACTH e do cortisol, ocorre a ativação do sistema nervoso simpático com conseqüente elevação da glicemia, pressão arterial sanguínea e freqüência cardíaca. O CRH não deve ser o único neurohormônio do eixo HHA envolvido na resposta ao estresse, existindo evidências de que a arginina-vasopressina (AVP) de origem paraventricular também se eleva em resposta ao estresse 10,11 e age sinergicamente com o CRH, aumentando a secreção de ACTH. (Figura 1)

Alguns trabalhos em humanos falharam em demonstrar a ação inibitória do CRH na liberação de gonadotrofinas. ${ }^{12}$ Tal fato se deve, provavelmente, a dificuldades metodológicas de se reproduzir a ação direta do CRH no sistema nervoso central, uma vez que a administração periférica é inapropriada para alcançar concentrações fisiológicas nesse nível.

A seguir, será abordado como essas modificações no eixo HHA, produzidas pelo estresse, determinam alterações no funcionamento gonadal e na função reprodutiva. 
Resposta central ao estresse com ativação do eixo hipotálamo-hipófise-adrenal e inibição do eixo hipotálamohipófise-ovariano.

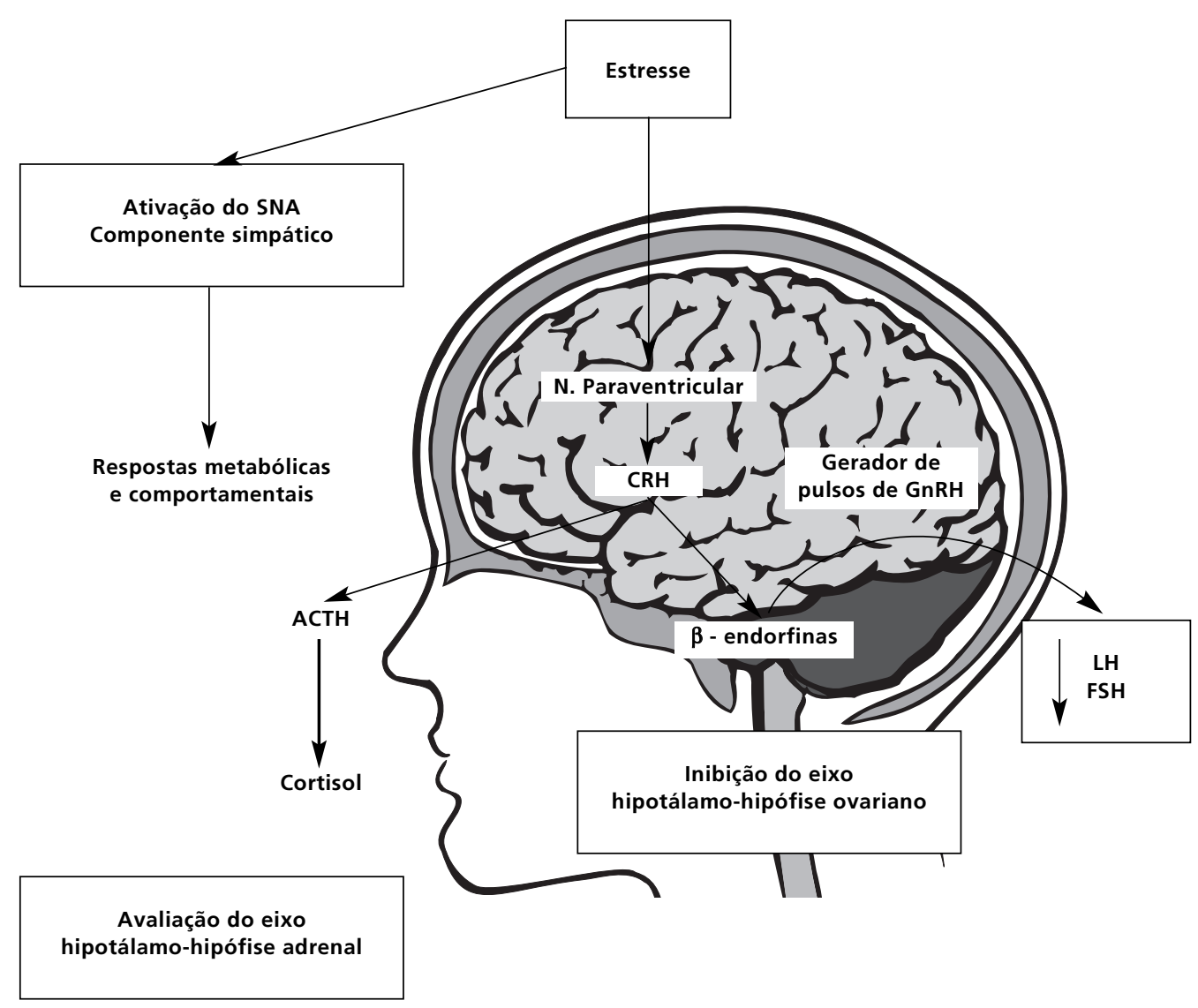

Fonte: Modificado de Ferrin, 1999.9

SNA = sistema nervoso autônomo; CRC = hormônio liberador de corticotrofina; GnRH = hormônio liberador de gonadotrofinas; $\mathrm{LH}=$ hormônio luteinizante; $\mathrm{FSH}=$ hormônio folículo estimulante; $\mathrm{ACTH}=$ hormônio

adrenocorticotrófico

\section{Eixo hipotálamo-hipófise-ovariano (HHO)}

Sabe-se que a secreção de GnRH pelo hipotálamo não se processa de forma tônica, mas sob a forma de pulsos que duram vários minutos, com intervalos variáveis de uma a três horas. Essa natureza pulsátil da secreção do GnRH é fundamental para o estímulo adequado para secreção das gonadotrofinas. Em conseqüência, observa-se um perfil também pulsátil na secreção das gonadotrofinas, em especial na do LH. 10,13

Na prática clínica, é comum a ocorrência de casos de irregularidade menstrual ou anovulação /infertilidade, desencadeados ou concomitantes a um evento estressante. Nesses casos, a inibição da pulsatilidade do GnRH pelo estresse e conseqüente inibição da função hipofisária-gonadal normal parece ser o fator causal. Conforme descrito anteriormente, a ativação do eixo hipotálamo-hipófise-adrenal também desempenha um papel importante, interferindo no eixo hipotálamo-hipófise-ovariano de forma predominantemente inibitória.

O núcleo arqueado, localizado no hipotálamo tuberal e principal local de produção de GnRH, recebe vários circuitos neuronais do sistema límbico (responsável pelas emoções), que modificam a inten- 
sidade e a freqüência dos pulsos de GnRH, explicando, assim, as várias formas de alterações menstruais observadas em mulheres submetidas a fortes impactos emocionais. $\mathrm{Na}$ dependência da intensidade e duração desses estímulos, tais mulheres podem, inclusive, desenvolver "amenorréia hipotalâmica funcional" ou "anovulação crônica hipotalâmica", caracterizada pela quiescência ovariana, amenorréia e conseqüente infertilidade.

Conforme já destacado, essa síndrome também pode estar associada com outras variáveis referentes ao estilo de vida, como perda de peso ou distúrbio alimentar e prática de exercícios físicos em excesso. Na grande maioria das vezes, o que se observa é a ocorrência de distúrbios menstruais do tipo oligoamenorréia, em geral autolimitados, refletindo a relação com os fenômenos psicológicos que o motivaram, muitas vezes já resolvidos por ocasião do atendimento médico. 9

Existe uma relação estreita entre o evento estressor e o desenvolvimento da amenorréia. Contudo, na prática, é difícil estabelecer a interferência do estresse sobre o ciclo menstrual. Cada mulher responde de forma diversa diante de um mesmo estímulo estressor, em virtude das características individuais e das diferentes estratégias de adaptação desenvolvidas.

Mulheres com a síndrome de anovulação crônica hipotalâmica podem apresentar diminuição na freqüência dos pulsos de LH associada a elevação dos níveis séricos de cortisol, refletindo aumento de atividade do CRH. ${ }^{14}$ Esse estudo sugere que, pelo menos em um subgrupo de pacientes com amenorréia, o eixo HHA está ativado, demonstrando a relação amplamente estudada em animais, entre esse eixo e o HHO.

$\mathrm{O}$ efeito do estresse no eixo HHO depende ainda das concentrações de estrógeno. Na presença de concentrações elevadas de estrógenos, a ativação do eixo HHA estimula a liberação de LH, de modo que um estresse agudo na fase folicular do ciclo menstrual pode desencadear pulsos precoces de $\mathrm{LH}$, interferindo na maturação do folículo e na ovulação. 15 Além das alterações do eixo HHA, os peptídeos opióides endógenos (particularmente as beta-endorfinas) também desempenham importante função inibitória do eixo HHO, em situações de estresse.

\section{Estresse e infertilidade. Causa ou conseqüência?}

A função reprodutiva humana depende de complexas interações entre o sistema nervoso central (SNC), hipófise, ovários, outras estruturas endócrinas e órgãos reprodutivos. Para que ocorra ciclicidade menstrual, é necessário que haja uma função ovulatória regular, o que depende, além da integridade anatômica das diversas estruturas do eixo reprodutivo, de uma sincronia entre suas ações.

A função cíclica ovariana pode ser facilmente perturbada por um estresse emocional, levando à interrupção temporária das menstruações. Sabe-se, por exemplo, que o desejo obsessivo de engravidar pode desencadear amenorréia temporária, dificultando, ainda mais, a concepção. Entretanto, é importante considerar as diferenças individuais na resposta a um determinado evento estressor, conforme discutido anteriormente.

Existem vários sistemas regulatórios, que operam por meio de conexões nervosas, neurotransmissores e hormônios, que influenciam os mecanismos reprodutivos. Pesquisas destacam que os agentes estressores reduzem a fertilidade pelas influências que causam nos mecanismos que regulam os eventos da fase folicular do ciclo menstrual.10 A ativação do eixo hipotálamo-hipófise-adrenal induzida pelo estresse, reduziria a pulsatilidade do GnRH, privando o folículo ovariano de adequado suporte de gonadotrofinas e resultando em anovulação.

No momento do crescimento folicular, o oócito mantém contato direto com as células da granulosa, através da zona pelúcida. Assim, os eventos que influenciam a integridade da função folicular podem ter efeitos diretos na viabilidade do oócito. Conseqüentemente, algum evento que venha alterar a atividade das células da granulosa pode também influenciar as taxas de gravidez. ${ }^{16}$

Nesse sentido, diferentes mecanismos biológicos relacionados ou desencadeados pelo estresse podem alterar a função reprodutiva a ponto de causar redução da fertilidade. No entanto, é extremamente difícil estabelecer relações de causa-efeito, posto que as causas de infertilidade são múltiplas e não envolvem apenas fatores ligados à fisiologia feminina mas, também, causas masculinas de infertilidade.

É conveniente mencionar que os pesquisadores estão divididos na proposição de que a infertilidade pode ter causas psicológicas (hipótese psicogênica), ou ser causa de uma variedade de dificuldades psicológicas.5,17-19

Historicamente, os estudos psicológicos sobre infertilidade focalizavam primariamente a mulher, sobre a qual se pensava que era hostil e muito dependente de sua mãe ou que agressivamente imitava o papel masculino. Também se sustentava que a mulher infértil era conflitante e ambivalente acerca da maternidade, o que resultava na sua incapacidade 
de conceber. ${ }^{20}$ Esse modelo psicogênico foi dominante nos idos de 1980, período em que pesquisadores afirmaram que a maioria dos estudos envolvendo mulheres inférteis foi voltada mais para a abordagem dos fatores psicológicos na etiologia do problema do que para as conseqüências psicológicas da infertilidade. 17

A partir de então, os estudos mais recentes se preocuparam mais com a idéia de que a infertilidade pode ser origem de significativo estresse psicológico, em virtude de esse problema ser considerado por muitas mulheres inférteis como o mais estressante evento de suas vidas.7,18 Valoriza-se, ainda, o estresse originado do tratamento da infertilidade, pelo fato dos casais estarem expostos a grande quantidade de informações e diversos tipos de intervenções e novos tratamentos médicos, através das tecnologias de reprodução assistida (RA). 19

Vários estudos sugerem que o estresse pode influenciar negativamente o resultado do tratamento da infertilidade e a prática clínica confirma a presença de sintomas psicológicos determinados pelo tratamento, como ansiedade, estresse, isolamento familiar/social e dificuldades sexuais. $2,5,7$

$\mathrm{Na}$ verdade, é mais plausível admitir que as duas hipóteses se completem, uma vez que os efeitos do estresse sobre o eixo HHO, levando à quadros anovulatórios, não podem ser ignorados, nem tampouco podem ser negligenciadas as conseqüências psicológicas advindas do quadro de infertilidade.

\section{Importância da abordagem psicológica em serviços de reprodução humana}

A teoria biopsicossocial da infertilidade proposta por Gerrity 21 concebe a infertilidade como acontecimento humano no qual estão comprometidas uma pessoa (homem ou mulher) e algumas relações (dos cônjuges entre si, com a equipe médica e com o contexto social no qual estão inseridos. Nessa perspectiva, o psicólogo pode desempenhar papel importante auxiliando os casais na elaboração dos conflitos psicológicos desencadeados ou agravados pela infertilidade. A atuação do psicólogo deve estar associada ao tratamento médico, avaliando como o diagnóstico, a sugestão de tratamento e os pedidos de exames estão sendo entendidos pelo casal e identificando as dificuldades psicológicas que possam afetar a evolução do tratamento.21-24

A literatura descritiva apresenta a infertilidade como uma experiência devastadora, comparando-a ao divórcio e ao diagnóstico de uma doença crônica grave.18 Além disso, muitas mulheres inférteis sentem-se desvalorizadas, anormais, incompletas e insatisfeitas. 22,25

Diversos trabalhos apontam que mulheres inférteis expressam sentimentos de frustração e inadequação, ${ }^{17}$ perda de auto-estima, ${ }^{26}$ além de depressão e ansiedade, 18 podendo gerar sentimentos nos familiares do casal e na equipe médica. 27

$\mathrm{O}$ tratamento da infertilidade implica em extensos exames diagnósticos e tratamentos médicos de longa duração, que podem precipitar sintomas psicológicos, sendo de maior freqüência a ansiedade e a depressão, além da raiva, frustração, isolamento familiar/social e dificuldades sexuais. $2,17,21,23$ Esses sintomas afetam entre 25 a $60 \%$ das pessoas inférteis. A ansiedade costuma aparecer pela natureza estressante dos tratamentos e pelo medo de não conceber, estando a depressão mais relacionada ao resultado negativo do tratamento. 26

Caso estejam instalados os transtornos psicológicos, faz-se necessária uma ação terapêutica, na qual o psicólogo pode utilizar terapia individual, de casal ou de grupo, dependendo da sintomatologia apresentada. Na terapia de casal, os objetivos variam de acordo com as dificuldades em aceitar a infertilidade e o seu tratamento, uma vez que a ocorrência da infertilidade pode atingir o homem e a mulher de forma distinta. Geralmente, as mulheres ficam mais preocupadas com a situação, querendo logo iniciar o tratamento. Os homens, em geral, mais passivos, encontram maiores dificuldades em expressar seus sentimentos. 25 Esse conflito no equilíbrio entre os próprios desejos e aqueles do companheiro, seria o núcleo da terapia de casal.

Os pacientes inférteis que participam de um grupo terapêutico utilizam uma mesma linguagem e partilham as mesmas vivências, o que pode propiciar, em curto prazo, uma melhor adaptação ao tratamento da infertilidade, possibilitando-os a aceitar e a assumir o seu problema, de forma menos conflituosa. Além disso, o grupo terapêutico pode conter e absorver as angústias e dúvidas dos participantes, propiciando um estímulo às capacidades positivas e minimizando sentimentos de isolamento e estigma social. $20 \mathrm{O}$ atendimento em grupo para o casal infértil pode desenvolver-se tanto com o casal quanto com apenas um dos cônjuges, dependendo das necessidades de cada um.

Embora a infertilidade conjugal seja um problema de natureza médica, psicológica e social, alguns profissionais a tratam como uma doença estritamente orgânica, desconsiderando a interação dos aspectos biológicos e psicológicos.

A função do psicólogo, portanto, é conscientizar o casal infértil da magnitude dos seus problemas 
dentro do contexto biopsicossocial, ajudando-o a enfrentar mais adequadamente determinadas situações conflitivas inerentes à infertilidade e procurar integrá-lo à equipe profissional dos serviços de medicina reprodutiva.

\section{Conclusões}

Existe uma relação significativa entre o estresse e a função reprodutiva, visto que os eventos estressores afetam o eixo hipotálamo-hipófise-ovariano através de diversos mecanismos biológicos, explicando assim, as várias formas de alterações do ciclo menstrual observadas em mulheres submetidas a fortes impactos emocionais. Por outro lado, os procedimentos envolvidos no diagnóstico e tratamento da infertilidade podem exacerbar problemas psicológicos. Nesse sentido, enfatiza-se a importância da abordagem psicológica nos Serviços de Infertilidade e Reprodução Assistida, a qual deve ser instituída desde as etapas iniciais do acompanhamento clínico.

\section{Referências}

1. Warren MP, Fried JL. Hypothalamic amenorrhea. The effects of environmental stresses on the reproductive system: a central effect of the central nervous system. Endocrinol Metab Clin North Am 2001; 30:611-29.

2. Newton CR, Sherrard W, Glavac I. The fertility problem inventory: measuring perceived infertility-related stress. Fertil Steril 1999; 72: 54-62.

3. O' Moore AM, O' Moore RR, Harrison RF, Murphy G, Carruthers ME. Psychosomatic aspects in idiopathic infertility: effects of treatment with autogenic training. J Psychosom Res 1983; 27: 145-51.

4. Armario A, Marti O, Molina T, Pablo J, Valdes M. Acute stress markers in humans: response of plasma glucose, cortisol and prolactin to two examinations differing in anxiety they provoke. Psychoneuroendocrinology 1996; 21 $17-24$.

5. Domar AD, Clapp D, Slawsby E, Dusek J, Kessel B Freizinger M. Impact of group psychological interventions on pregnancy rates in infertility women. Fertil Steril 2000; 73: $805-11$.

6. Gallinelli A, Roncaglia R, Matteo ML, Ciaccio I, Volpe A, Facchinetti F. Immunological changes and stress are associated with different implantation rates in patients undergoing in vitro fertilization-embryo transfer. Fertil Steril 2001; 76: 85-91

7. Cohen HK, Chu E, Natarajan L, Sieber W. A prospective study of stress among women undergoing in vitro fertilization or gamete intrafallopian transfer. Fertil Steril 2001; 76: 675-87.

8. Urdampilleta L, Fernandez D. Psicologia da anovulação. In: Busso NE, Acosta AA, Remohi J. Indução da ovulação. São Paulo: Atheneu; 1999.

9. Ferin M. Stress and the reproductive cycle. J Clin Endocrinol Metab 1999; 84:1768-74.

10. Dobson H, Smith RF. What is stress, and how does it affect reproduction? Anim Reprod Sci 2000; 61: 743-52.

11. Herman JP, Cullinan WE. Neurocircuitry of stress: central control of the hypothalamo-pituitary-adrenocortical axis. Trends Neurosci 1997; 20: 78-84.

12. Barbarino A, De Marinis L, Folli G, Tofani A, Della Casa S, D'Amico C, Mancini A, Corsello SM, Sambo P, Barini A. Corticotropin-releasing hormone inhibition of gonadotropin secretion during the menstrual cycle. Metabolism 1989; 38:504-6.

13. Smith RF, Dobson H. Hormonal interactions within the hypothalamus and pituitary with respect to stress and reproduction in sheep. Domest Anim Endocrinol 2002; 23: 7585 .

14. Biller BM, Federoff HJ, Koenig JI, Klibanski A. Abnormal cortisol secretion and responses to corticotropin-releasing hormone in women with hypothalamic amenorrhea. J Clin Endocrinol Metab 1990; 70: 311-7.

15. Puder JJ, Freda PU, Goland RS, Ferin M, Wardlaw SL. Stimulatory effects of stress on gonadotropin secretion in estrogen-treated women. J Clin Endocrinol Metab 1999; 85: 2184-88.

16. Macéa JR, Macéa MI, Busso NE. Função ovariana. In: Busso NE, Acosta AA, Remohi J. Indução da ovulação. São Paulo: Atheneu; 1999.

17. Bell JS. Psychological problems among patients attending an infertility clinic. J Psychosom Res 1980; 25: 1-3.

18. Connolly KJ, Edelmann RJ, Cooke ID, Robson J. The impact of infertility on psychological functioning. J Psychosom Res 1992; 36: 459-68.

19. Facchinetti F, Matteo ML, Artini GP, Volpe A, Genazzani AR. An increased vulnerability to stress is associated with a poor outcome of in vitro fertilization-embryo transfer treatment. Fertil Steril 1997; 67: 309-14.

20. Burns LH, Covington SN. Psychology of infertility. In: Burns LH, Covington SN. Infertility counseling-a comprehensive handbook for clinicians. New York: Parthenon; 2000. p. 3-25. 
21. Gerrity DA. A biopsychosocial theory of infertility. Fam J 2001; 9: 151-8.

22. Greil AL. Infertility and psychological distress: a critical review of the literature. Soc Sci Med 1997; 45: 1679-704.

23. Daniluk JC. Infertility: intrapersonal and interpersonal impact. Fertil Steril 1988; 49: 982-90.

24. Eriksen K. Infertility and the search for family. Fam J 2001; 9: 55-61.

25. Trindade ZA, Enumo SRF. Triste e incompleta: uma visão feminina da mulher infértil. Psicol USP 2002; 13: 1-18.
26. Guerra D, Llobera A, Veiga A, Barri PN. Psychiatric morbidity in couples attending a fertility service. Hum Reprod 1988; 13: 1733-36.

27. Daniluk JC. If we had it to do over again. Couples reflections on their experiences of infertility treatments. Fam J 2001; 9: 122-33.

28. Applegarth LD. Individual counseling and psychotherapy. In: Burns LH, Covington SN. Infertility counseling-a comprehensive handbook for clinicians. New York: Parthenon; 2000. p. 85-101.

Recebido em 10 de março de 2004

Versão final apresentada em 26 de agosto de 2004

Aprovado em 28 de setembro de 2004 